A Bibliometric Analysis of the 100 Most Cited Articles on Problem-based Learning in Medical Education

Violet Kulo, EdD, MS, MA

Associate Professor, Health Professions Education

Graduate School

University of Maryland, Baltimore

ORCID: 0000-0002-5386-9549

Christina Cestone, $\mathrm{PhD}, \mathrm{MA}$

Executive Director, Faculty Center for Teaching and Learning

Associate Professor, Health Professions Education

Graduate School

University of Maryland, Baltimore

ORCID: 0000-0001-8960-0026 


\title{
A Bibliometric Analysis of the 100 Most Cited Articles on Problem-based Learning in Medical Education
}

\begin{abstract}
Introduction

Problem-based learning (PBL) is an instructional approach used in medical education that is characterized by solving patient-based problems in small groups with tutor guidance. More than 50 years since PBL's inception, many questions remain to be addressed about its processes and learning outcomes. This study examined the characteristics of the 100 most cited articles on PBL in medical education to identify landmark papers that have made significant contributions to PBL research.
\end{abstract}

\section{Methods}

The authors conducted a bibliometric analysis of the most cited articles on PBL in medical education. Results were systematically reviewed for citation frequency, publication year, journal, article type, article focus, authors, and country of first author at the time of publication.

\section{Results}

The articles were contributed by 212 authors in 23 journals between 1981-2016. Most articles (68\%) were published in Medical Education, Academic Medicine, and Medical Teacher. The majority of the articles (71\%) originated from Netherlands, Canada, and the United States and six prolific authors were identified. Almost half of the articles are classified as empirical research. Article foci included theoretical foundations of PBL, curriculum design, learning outcomes and processes, tutors, assessment, guides to PBL implementation, commentaries, and student well-being.

\section{Discussion}


The top-cited articles cover a broad range of topics from the theoretical basis for PBL to its effectiveness. The strong author and country collaborative networks indicate continued global interest in the PBL instructional method. Our findings show a paucity of studies on the actual implementation of PBL, its long-term impact, as well as rigorous qualitative studies.

Keywords: bibliometric analysis, problem-based learning, medical education

\section{Introduction}

In the 1992-93 Annual Medical School Questionnaire administered by the Liaison Committee on Medical Education (LCME), 109 out of 125 (87\%) medical schools in the United States (US) reported that they used problem-based learning (PBL) in their curriculum [1]. A little over a decade later, a study conducted to examine the prevalence of PBL in the preclinical curriculum found that 86 out of 123 (70\%) US medical schools used PBL [2]. More recently, the 2019-2020 Curriculum Inventory data reported annually to the Association of American Medical Colleges (AAMC) shows that 68 out of 153 (44\%) medical schools in US and Canada use PBL [3]. Problem-based learning as an instructional approach in medical schools has a long history of research and debate about its theoretical origins, processes, and learning outcomes [4-12].

Problem-based learning originated from McMaster University in Canada in the 1960s as a solution to traditional medical education problems that inhibited clinical reasoning ability $[13,14]$. Barrows and Tamblyn [15] defined PBL as "the learning that results from the process of working toward the understanding or resolution of a problem" (p. 1). The authors noted that the main objectives of PBL are for students to construct an integrated body of knowledge related to the problem and to develop or apply problem-solving skills. While there are many variations in 
how PBL is implemented in medical schools, Barrows [13] described the core model of PBL, which includes six characteristics. First, learning should be student-centered. The second characteristic is that learning needs to occur in small groups. Third, a tutor acts as a facilitator or guide for the group. Fourth, authentic problems are presented first to provide the relevance and motivation for learning. Fifth, learners construct knowledge and develop clinical problemsolving skills by engaging with the problem. Lastly, new knowledge is acquired through selfdirected learning.

The theoretical basis of PBL has been discussed by several researchers. For example, Schmidt [11] contended that PBL is based on the information processing theory and described the extent to which it meets three principles that facilitate knowledge acquisition including, activating students' relevant prior learning, providing contexts that are similar to real-world settings, and affording opportunities for students to elaborate on their knowledge. Similarly, Dolmans et al. [16] argued that PBL is based on four key learning principles: constructive, selfdirected, collaborative, and contextual. The evidence for the effectiveness of PBL over traditional instructional methods is inconclusive. A number of studies found evidence for the positive effects of PBL on student learning [17-19], whereas other studies found no consistent evidence in favor of PBL [20-23].

In a synthesis of meta-analyses and systematic reviews of PBL research, Hung and colleagues [24] identified three main waves in the research over 50 years. The three waves were: "Does PBL work?," "How does PBL work?," and "How does PBL work in different specific contexts?" The authors called for the next wave of research to focus on why PBL works or does not work. As the fourth wave of research continues, it is important to identify the most influential 
articles on PBL in the 50-year period since its inception to guide researchers on the landmark papers that have made significant contributions to PBL research in medical education.

Therefore, we conducted a bibliometric analysis of the highly cited articles on PBL in medical education. Bibliometrics is the quantitative study of publications to measure and describe the output of research in a field $[25,26]$. Several researchers have conducted bibliometric analyses in medical education to analyze types of empirical studies published in the top journals [27], examine highly cited articles [28,29], characterize the literature on validation of assessments [30], and explore the landscape of knowledge syntheses [31].

The most recent bibliometric analysis on PBL published in 2017 analyzed the characteristics of the most frequently cited articles on PBL across all disciplines [32]. Our interest in completing a PBL bibliometric analysis in medical education was to not only analyze the bibliometric characteristics of the 100 most cited articles to understand the state of knowledge in the field, but also to identify landmark papers and provide a resource for researchers new to PBL research in medical education.

\section{Methods}

We followed guidelines for conducting a bibliometric analysis as described by Donthu and colleagues [33]. The steps are: define the aims and scope of the bibliometric study, choose the techniques for bibliometric analysis, collect the data, run the analysis including performance analysis and science mapping, and report the findings.

For the initial search, the authors searched Scopus in the title, abstract, and keywords using the following search terms: 'problem-based learning', 'pbl', 'problem based learning', 'problem-based curriculum', 'problem centered curriculum', 'problem-based instruction', 
'problem-oriented instruction,' AND 'med*'. We sorted the search results in Scopus in descending order using the "Sort on" option "Cited by (highest)."

Inclusion and exclusion criteria

Articles that were eligible for inclusion were those whose focus was PBL in medical education and were in the English language. Exclusion criteria included articles that were about PBL in other disciplines such as engineering, computer science, and physics, and non-English language articles. Articles on PBL that were published in books, conference proceedings, and editorials were also excluded. The authors independently reviewed the titles and abstracts of the articles and selected the ones that met the inclusion criteria.

Data collection

Both authors listed the results in a Microsoft Excel spreadsheet in descending order based on the number of citations. The spreadsheet was organized in 9 columns: rank, article title, authors, number of citations, publication year, name of journal, article type (descriptive, research, review, or theoretical), article focus, and country of first author at the time of publication. For this study, we defined descriptive articles as papers that described PBL, its characteristics, and implementation in the classroom. Research articles were defined as studies that utilized quantitative, qualitative, or mixed methods to investigate the implementation of PBL in order to make valid conclusions about the effectiveness, effects, or impact of PBL on student learning. Review articles were defined as papers that critically analyzed or synthesized recent or current literature on PBL and summarized the state and progress of research in PBL. Using Grant and Booth's [34] descriptions of the common types of reviews, we categorized reviews into narrative, meta-analysis, and systematic reviews. Theoretical articles were defined as articles that examined or described the underlying theoretical foundations of PBL. 
We met to review the independent lists and discussed differences in ranking to reach a consensus on inclusion or exclusion of the article. In the first round of review, we excluded eight articles whose focus was not PBL, and we were unsure about three articles from reading the abstracts only. For these three articles, we read the full articles before determining whether they should be included, which led to excluding two more of the three articles. The interrater reliability was $\kappa=0.928(p<.001)$, which fell in the almost perfect agreement range [35].

Next, we cross-checked the references of the five most highly cited review articles and realized that we had missed some articles because our search terms were not broad enough. We expanded our search terms and conducted a second search in which we excluded the term 'med*' and added the following terms to the initial search terms: 'problem-based', 'problem based', 'problem-solving', and 'problem solving'. We again reviewed the titles and abstracts of the articles independently and selected eligible articles that we missed in round one review. The second search added 18 new articles to the list. We reviewed Scopus for the final time on May 31, 2021 to update the number of citations for each article.

We reviewed 1,557 articles to arrive at the top 100 articles. This represented $5.3 \%$ of the total results returned in Scopus for the search $(n=29,521)$. Once the full list was compiled, we compared the citation rankings to ensure both common ranking position and total reference count.

Data analysis

The data screening process required ensuring consistency in the authors' names and removing errors and/or adding omissions in the author affiliations and countries. For example, "Norman GR" and "Norman G" referred to the same author, and thus, we checked all the authors' names to ensure the initials for the same authors were consistent. Analyses were 
conducted using Excel to calculate descriptive statistics, IBM SPSS Statistics [36] to compute Pearson correlation coefficient and Cohen's kappa coefficient, and VOSviewer [37] to create bibliometric network visualizations. In addition to citation frequency, we computed the following: publication years, authors, author network, country of publication network, publication journal frequency and journal impact factor, and article type.

\section{Results}

The 100 most cited articles in PBL in medical education are ranked in Table 1 in descending order by number of citations and by average citations per year for articles with the same absolute number of citations. 
Table 1. The 100 Most Cited Articles on Problem-based Learning in Medical Education

\begin{tabular}{|c|c|c|c|c|c|c|c|}
\hline Rank & Article Title & $\begin{array}{l}\text { First Author, } \\
\text { Year }\end{array}$ & $\begin{array}{l}\text { Number of } \\
\text { Citations }\end{array}$ & $\begin{array}{l}\text { Average } \\
\text { Citations } \\
\text { per Year }\end{array}$ & Journal & Article Type & $\begin{array}{l}\text { First Author's } \\
\text { Country at time of } \\
\text { Publication }\end{array}$ \\
\hline 1 & $\begin{array}{l}\text { Why minimal guidance during instruction does not work: An } \\
\text { analysis of the failure of constructivist, discovery, problem- } \\
\text { based, experiential, and inquiry-based teaching }\end{array}$ & $\begin{array}{l}\text { Kirschner, } \\
2006[7]\end{array}$ & 2997 & 199.8 & $\begin{array}{l}\text { Educational } \\
\text { Psychologist }\end{array}$ & Theoretical & Netherlands \\
\hline 2 & Problem-Based Learning: What and how do students learn? & $\begin{array}{l}\text { Hmelo-Silver, } \\
2004[40]\end{array}$ & 1938 & 114 & $\begin{array}{l}\text { Educational } \\
\text { Psychology } \\
\text { Review }\end{array}$ & Review & USA \\
\hline 3 & $\begin{array}{l}\text { Problem-based learning: A review of literature on its outcomes } \\
\text { and implementation issues }\end{array}$ & $\begin{array}{l}\text { Albanese, } 1993 \\
{[20]}\end{array}$ & 1663 & 59.4 & $\begin{array}{l}\text { Academic } \\
\text { Medicine }\end{array}$ & Review & USA \\
\hline 4 & $\begin{array}{l}\text { Scaffolding and achievement in problem-based and inquiry } \\
\text { learning: A response to Kirschner, Sweller, and Clark (2006) }\end{array}$ & $\begin{array}{l}\text { Hmelo-Silver, } \\
2007[44]\end{array}$ & 1033 & 73.8 & $\begin{array}{l}\text { Educational } \\
\text { Psychologist }\end{array}$ & Descriptive & USA \\
\hline 5 & $\begin{array}{l}\text { The psychological basis of problem-based learning: A review of } \\
\text { the evidence }\end{array}$ & $\begin{array}{l}\text { Norman, } 1992 \\
{[10]}\end{array}$ & 1025 & 35.3 & $\begin{array}{l}\text { Academic } \\
\text { Medicine }\end{array}$ & Review & Canada \\
\hline 6 & A taxonomy of problem-based learning methods & $\begin{array}{l}\text { Barrows, } 1986 \\
{[53]}\end{array}$ & 1011 & 28.9 & $\begin{array}{l}\text { Medical } \\
\text { Education }\end{array}$ & Descriptive & Canada \\
\hline 7 & $\begin{array}{l}\text { Does problem-based learning work? A meta-analysis of } \\
\text { evaluative research }\end{array}$ & $\begin{array}{l}\text { Vernon, } 1993 \\
{[19]}\end{array}$ & 924 & 33 & $\begin{array}{l}\text { Academic } \\
\text { Medicine }\end{array}$ & Review & USA \\
\hline 8 & Effects of problem-based learning: A meta-analysis & $\begin{array}{l}\text { Dochy, } 2003 \\
{[17]}\end{array}$ & 819 & 45.5 & $\begin{array}{l}\text { Learning and } \\
\text { Instruction }\end{array}$ & Review & Netherlands \\
\hline 9 & $\begin{array}{l}\text { Problem-based learning in medicine and beyond: A brief } \\
\text { overview }\end{array}$ & $\begin{array}{l}\text { Barrows, } 1996 \\
{[13]}\end{array}$ & 715 & 28.6 & $\begin{array}{l}\text { New } \\
\text { Directions for } \\
\text { Teaching and } \\
\text { Learning }\end{array}$ & Descriptive & Canada \\
\hline 10 & Problem-based learning: rationale and description & $\begin{array}{l}\text { Schmidt, } 1983 \\
{[11]}\end{array}$ & 693 & 18.2 & $\begin{array}{l}\text { Medical } \\
\text { Education }\end{array}$ & Descriptive & Canada \\
\hline 11 & $\begin{array}{l}\text { Effectiveness of problem-based learning curricula: Research and } \\
\text { theory }\end{array}$ & $\begin{array}{l}\text { Colliver, } 2000 \\
{[56]}\end{array}$ & 611 & 29.1 & $\begin{array}{l}\text { Academic } \\
\text { Medicine }\end{array}$ & Descriptive & Canada \\
\hline
\end{tabular}




\begin{tabular}{|c|c|c|c|c|c|c|c|}
\hline 12 & Problem based learning & $\begin{array}{l}\text { Wood, } 2003 \\
{[57]}\end{array}$ & 532 & 29.6 & $\begin{array}{l}\text { British } \\
\text { Medical } \\
\text { Journal }\end{array}$ & Descriptive & UK \\
\hline 13 & $\begin{array}{l}\text { Effectiveness of problem-based learning curricula: Theory, } \\
\text { practice and paper darts }\end{array}$ & $\begin{array}{l}\text { Norman, } 2000 \\
{[45]}\end{array}$ & 444 & 21.1 & $\begin{array}{l}\text { Medical } \\
\text { Education }\end{array}$ & Descriptive & Canada \\
\hline 14 & $\begin{array}{l}\text { Effects of problem-based learning: A meta-analysis from the } \\
\text { angle of assessment }\end{array}$ & $\begin{array}{l}\text { Gijbels, } 2005 \\
{[6]}\end{array}$ & 432 & 27 & $\begin{array}{l}\text { Review of } \\
\text { Educational } \\
\text { Research }\end{array}$ & Review & Netherlands \\
\hline 15 & Foundations of problem-based learning: some explanatory notes & $\begin{array}{l}\text { Schmidt, } 1993 \\
{[12]}\end{array}$ & 423 & 15.1 & $\begin{array}{l}\text { Medical } \\
\text { Education }\end{array}$ & Theoretical & Netherlands \\
\hline 16 & $\begin{array}{l}\text { Problem-based learning: Future challenges for educational } \\
\text { practice and research }\end{array}$ & $\begin{array}{l}\text { Dolmans, } 2005 \\
{[16]}\end{array}$ & 417 & 26.1 & $\begin{array}{l}\text { Medical } \\
\text { Education }\end{array}$ & Descriptive & Netherlands \\
\hline 17 & Problem-based learning: Have the expectations been met? & $\begin{array}{l}\text { Berkson, } 1993 \\
{[58]}\end{array}$ & 327 & 11.7 & $\begin{array}{l}\text { Academic } \\
\text { Medicine }\end{array}$ & Descriptive & Canada \\
\hline 18 & The process of problem-based learning: What works and why & $\begin{array}{l}\text { Schmidt, } 2011 \\
{[59]}\end{array}$ & 324 & 32.4 & $\begin{array}{l}\text { Medical } \\
\text { Education }\end{array}$ & Descriptive & Netherlands \\
\hline 19 & $\begin{array}{l}\text { Simulation-based training is superior to problem-based learning } \\
\text { for the acquisition of critical assessment and management skills }\end{array}$ & $\begin{array}{l}\text { Steadman, } \\
2006[60]\end{array}$ & 316 & 21.1 & $\begin{array}{l}\text { Critical Care } \\
\text { Medicine }\end{array}$ & Research & USA \\
\hline 20 & $\begin{array}{l}\text { The effects of problem-based learning during medical school on } \\
\text { physician competency: A systematic review }\end{array}$ & Koh, $2008[18]$ & 315 & 24.2 & $\begin{array}{l}\text { Canadian } \\
\text { Medical } \\
\text { Association } \\
\text { Journal } \\
\end{array}$ & Review & Singapore \\
\hline 21 & Learner centred approaches in medical education & $\begin{array}{l}\text { Spencer, } 1999 \\
\text { [61] }\end{array}$ & 315 & 14.3 & $\begin{array}{l}\text { British } \\
\text { Medical } \\
\text { Journal }\end{array}$ & Descriptive & UK \\
\hline 22 & $\begin{array}{l}\text { Self-directed learning in problem-based learning and its } \\
\text { relationships with self-regulated learning }\end{array}$ & $\begin{array}{l}\text { Loyens, } 2008 \\
{[8]}\end{array}$ & 289 & 22.2 & $\begin{array}{l}\text { Educational } \\
\text { Psychology } \\
\text { Review }\end{array}$ & Descriptive & Netherlands \\
\hline 23 & Essentials of problem-based learning & $\begin{array}{l}\text { Walton, } 1989 \\
{[62]}\end{array}$ & 259 & 8.1 & $\begin{array}{l}\text { Medical } \\
\text { Education } \\
\end{array}$ & Descriptive & UK \\
\hline 24 & $\begin{array}{l}\text { Promoting professional knowledge, experiential learning and } \\
\text { critical thinking for medical students }\end{array}$ & $\begin{array}{l}\text { Maudsley, } \\
2000[63]\end{array}$ & 245 & 11.7 & $\begin{array}{l}\text { Medical } \\
\text { Education }\end{array}$ & Descriptive & UK \\
\hline 25 & $\begin{array}{l}\text { Problem-based learning: Why curricula are likely to show little } \\
\text { effect on knowledge and clinical skills }\end{array}$ & $\begin{array}{l}\text { Albanese, } 2000 \\
\text { [4] }\end{array}$ & 243 & 11.6 & $\begin{array}{l}\text { Medical } \\
\text { Education }\end{array}$ & Theoretical & USA \\
\hline
\end{tabular}




\begin{tabular}{|c|c|c|c|c|c|c|c|}
\hline 26 & $\begin{array}{l}\text { Do we all mean the same thing by 'problem-based learning'? A } \\
\text { review of the concepts and a formulation of the ground rules }\end{array}$ & $\begin{array}{l}\text { Maudsley, } \\
1999[41]\end{array}$ & 233 & 10.6 & $\begin{array}{l}\text { Academic } \\
\text { Medicine }\end{array}$ & Review & UK \\
\hline 27 & Facilitating collaborative knowledge building & $\begin{array}{l}\text { Hmelo-Silver, } \\
2008 \text { [64] }\end{array}$ & 231 & 17.8 & $\begin{array}{l}\text { Cognition and } \\
\text { Instruction }\end{array}$ & Research & USA \\
\hline 28 & $\begin{array}{l}\text { Comparing problem-based learning with case-based learning: } \\
\text { Effects of a major curricular shift at two institutions }\end{array}$ & $\begin{array}{l}\text { Srinivasan, } \\
2007[65]\end{array}$ & 228 & 16.3 & $\begin{array}{l}\text { Academic } \\
\text { Medicine }\end{array}$ & Research & USA \\
\hline 29 & $\begin{array}{l}\text { First year medical student stress and coping in a problem-based } \\
\text { learning medical curriculum }\end{array}$ & $\begin{array}{l}\text { Moffat, } 2004 \\
{[50]}\end{array}$ & 228 & 13.4 & $\begin{array}{l}\text { Medical } \\
\text { Education }\end{array}$ & Research & UK \\
\hline 30 & $\begin{array}{l}\text { Problem-based learning and medical education forty years on: A } \\
\text { review of its effects on knowledge and clinical performance }\end{array}$ & $\begin{array}{l}\text { Neville, } 2009 \\
{[66]}\end{array}$ & 226 & 17.4 & $\begin{array}{l}\text { Medical } \\
\text { Principles and } \\
\text { Practice }\end{array}$ & Review & Canada \\
\hline 31 & $\begin{array}{l}\text { The approaches to learning of students in a traditional and in an } \\
\text { innovative problem-based medical school }\end{array}$ & $\begin{array}{l}\text { Newble, } 1986 \\
{[67]}\end{array}$ & 221 & 6.3 & $\begin{array}{l}\text { Medical } \\
\text { Education }\end{array}$ & Research & Australia \\
\hline 32 & $\begin{array}{l}\text { What makes a tutor effective? a structural-equations modeling } \\
\text { approach to learning in problem-based curricula }\end{array}$ & $\begin{array}{l}\text { Schmidt, } 1995 \\
{[68]}\end{array}$ & 214 & 8.2 & $\begin{array}{l}\text { Academic } \\
\text { Medicine }\end{array}$ & Research & Netherlands \\
\hline 33 & $\begin{array}{l}\text { The New Mexico experiment: Educational innovation and } \\
\text { institutional change }\end{array}$ & $\begin{array}{l}\text { Kaufman, } 1989 \\
\text { [69] }\end{array}$ & 208 & 6.5 & $\begin{array}{l}\text { Academic } \\
\text { Medicine }\end{array}$ & Research & Canada \\
\hline 34 & $\begin{array}{l}\text { Problem-based learning is compatible with human cognitive } \\
\text { architecture: Commentary on Kirschner, Sweller, and Clark } \\
\text { (2006) }\end{array}$ & $\begin{array}{l}\text { Schmidt, } 2007 \\
{[70]}\end{array}$ & 203 & 14.5 & $\begin{array}{l}\text { Educational } \\
\text { Psychologist }\end{array}$ & Descriptive & Netherlands \\
\hline 35 & $\begin{array}{l}\text { Effect of problem-based, self-directed undergraduate education } \\
\text { on life-long learning }\end{array}$ & Shin, 1993 [71] & 203 & 7.3 & $\begin{array}{l}\text { Canadian } \\
\text { Medical } \\
\text { Association } \\
\text { Journal }\end{array}$ & Research & Canada \\
\hline 36 & $\begin{array}{l}\text { Fifteen years of experience with progress testing in a problem- } \\
\text { based learning curriculum }\end{array}$ & $\begin{array}{l}\text { van der } \\
\text { Vleuten, } 1996 \\
{[49]}\end{array}$ & 187 & 7.5 & $\begin{array}{l}\text { Medical } \\
\text { Teacher }\end{array}$ & Descriptive & Netherlands \\
\hline 37 & $\begin{array}{l}\text { Problem-solving skills, solving problems and problem-based } \\
\text { learning }\end{array}$ & $\begin{array}{l}\text { Norman, } 1988 \\
\text { [72] }\end{array}$ & 181 & 5.5 & $\begin{array}{l}\text { Medical } \\
\text { Education }\end{array}$ & Review & Canada \\
\hline
\end{tabular}




\begin{tabular}{|c|c|c|c|c|c|c|c|}
\hline 38 & $\begin{array}{l}\text { Problem based learning in continuing medical education: A } \\
\text { review of controlled evaluation studies }\end{array}$ & $\begin{array}{l}\text { Smits, } 2002 \\
{[23]}\end{array}$ & 175 & 9.2 & $\begin{array}{l}\text { British } \\
\text { Medical } \\
\text { Journal }\end{array}$ & Review & Netherlands \\
\hline 39 & $\begin{array}{l}\text { Constructivist, problem-based learning does work: A meta- } \\
\text { analysis of curricular comparisons involving a single medical } \\
\text { school }\end{array}$ & $\begin{array}{l}\text { Schmidt, } 2009 \\
{[73]}\end{array}$ & 172 & 14.3 & $\begin{array}{l}\text { Educational } \\
\text { Psychologist }\end{array}$ & Review & Netherlands \\
\hline 40 & $\begin{array}{l}\text { Comparing the effects of problem-based and conventional } \\
\text { curricula in an international sample }\end{array}$ & $\begin{array}{l}\text { Schmidt, } 1987 \\
{[74]}\end{array}$ & 168 & 4.9 & $\begin{array}{l}\text { Journal of } \\
\text { Medical } \\
\text { Education } \\
\text { (now } \\
\text { Academic } \\
\text { Medicine) }\end{array}$ & Review & Netherlands \\
\hline 41 & $\begin{array}{l}\text { AMEE Medical Education Guide No. 15: Problem-based } \\
\text { learning: A practical guide }\end{array}$ & $\begin{array}{l}\text { Davis, } 1999 \\
{[46]}\end{array}$ & 163 & 7.4 & $\begin{array}{l}\text { Medical } \\
\text { Teacher }\end{array}$ & Descriptive & UK \\
\hline 42 & $\begin{array}{l}\text { The development of diagnostic competence: Comparison of a } \\
\text { problem-based, an integrated, and a conventional medical } \\
\text { curriculum }\end{array}$ & $\begin{array}{l}\text { Schmidt, } 1996 \\
\text { [75] }\end{array}$ & 159 & 6.4 & $\begin{array}{l}\text { Academic } \\
\text { Medicine }\end{array}$ & Research & Netherlands \\
\hline 43 & $\begin{array}{l}\text { Longterm effects of problem-based learning: A comparison of } \\
\text { competencies acquired by graduates of a problem-based and a } \\
\text { conventional medical school }\end{array}$ & $\begin{array}{l}\text { Schmidt, } 2006 \\
{[76]}\end{array}$ & 158 & 10.5 & $\begin{array}{l}\text { Medical } \\
\text { Education }\end{array}$ & Research & Netherlands \\
\hline 44 & $\begin{array}{l}\text { Differences between conventional and problem-based curricula } \\
\text { in their students' approaches to studying }\end{array}$ & $\begin{array}{l}\text { Coles, } 1985 \\
{[77]}\end{array}$ & 151 & 4.2 & $\begin{array}{l}\text { Medical } \\
\text { Education }\end{array}$ & Research & UK \\
\hline 45 & $\begin{array}{l}\text { Trends in research on the tutor in problem-based learning: } \\
\text { Conclusions and implications for educational practice and } \\
\text { research }\end{array}$ & $\begin{array}{l}\text { Dolmans, } 2002 \\
{[43]}\end{array}$ & 147 & 7.7 & $\begin{array}{l}\text { Medical } \\
\text { Teacher }\end{array}$ & Descriptive & Netherlands \\
\hline 46 & $\begin{array}{l}\text { Clinical problem-solving by medical students: a cross-sectional } \\
\text { and longitudinal analysis }\end{array}$ & $\begin{array}{l}\text { Neufeld, } 1981 \\
{[78]}\end{array}$ & 146 & 3.7 & $\begin{array}{l}\text { Medical } \\
\text { Education }\end{array}$ & Research & Canada \\
\hline 47 & Problem-based, self-directed learning & $\begin{array}{l}\text { Barrows, } 1983 \\
\text { [79] }\end{array}$ & 145 & 3.8 & $\begin{array}{l}\text { Journal of the } \\
\text { American } \\
\text { Medical } \\
\text { Association }\end{array}$ & Descriptive & Canada \\
\hline
\end{tabular}




\begin{tabular}{|c|c|c|c|c|c|c|c|}
\hline 48 & $\begin{array}{l}\text { Problem-based learning: Effects on the early acquisition of } \\
\text { cognitive skill in medicine }\end{array}$ & $\begin{array}{l}\text { Hmelo, } 1998 \\
{[80]}\end{array}$ & 144 & 6.3 & $\begin{array}{l}\text { Journal of the } \\
\text { Learning } \\
\text { Sciences }\end{array}$ & Research & USA \\
\hline 49 & $\begin{array}{l}\text { Effects of conventional and problem-based medical curricula on } \\
\text { problem solving }\end{array}$ & Patel, 1991 [81] & 144 & 4.8 & $\begin{array}{l}\text { Academic } \\
\text { Medicine }\end{array}$ & Research & Canada \\
\hline 50 & $\begin{array}{l}\text { Problem-based learning versus lecture-based learning in a course } \\
\text { of basic pharmacology: A controlled, randomized study }\end{array}$ & $\begin{array}{l}\text { Antephol, } 1999 \\
{[82]}\end{array}$ & 140 & 6.4 & $\begin{array}{l}\text { Medical } \\
\text { Education }\end{array}$ & Research & Germany \\
\hline 51 & $\begin{array}{l}\text { The effectiveness of problem-based learning compared to } \\
\text { traditional teaching in undergraduate psychiatry }\end{array}$ & $\begin{array}{l}\text { McParland, } \\
2004[83]\end{array}$ & 136 & 8.0 & $\begin{array}{l}\text { Medical } \\
\text { Education }\end{array}$ & Research & UK \\
\hline 52 & $\begin{array}{l}\text { Blended learning positively affects students' satisfaction and the } \\
\text { role of the tutor in the problem-based learning process: Results } \\
\text { of a mixed-method evaluation }\end{array}$ & $\begin{array}{l}\text { Woltering, } \\
2009[84]\end{array}$ & 135 & 11.3 & $\begin{array}{l}\text { Advances in } \\
\text { Health } \\
\text { Sciences } \\
\text { Education }\end{array}$ & Research & Germany \\
\hline 53 & $\begin{array}{l}\text { What do we know about cognitive and motivational effects of } \\
\text { small group tutorials in problem-based learning? }\end{array}$ & $\begin{array}{l}\text { Dolmans, } 2006 \\
{[85]}\end{array}$ & 133 & 8.9 & $\begin{array}{l}\text { Advances in } \\
\text { Health } \\
\text { Sciences } \\
\text { Education }\end{array}$ & Review & Netherlands \\
\hline 54 & $\begin{array}{l}\text { Solving problems with group work in problem-based learning: } \\
\text { Hold on to the philosophy }\end{array}$ & $\begin{array}{l}\text { Dolmans, } 2001 \\
{[86]}\end{array}$ & 132 & 6.6 & $\begin{array}{l}\text { Medical } \\
\text { Education }\end{array}$ & Descriptive & Netherlands \\
\hline 55 & $\begin{array}{l}\text { Helping students learn to think like experts when solving clinical } \\
\text { problems }\end{array}$ & $\begin{array}{l}\text { Mandin, } 1997 \\
{[87]}\end{array}$ & 132 & 5.5 & $\begin{array}{l}\text { Academic } \\
\text { Medicine }\end{array}$ & Descriptive & Canada \\
\hline 56 & $\begin{array}{l}\text { Does problem-based learning lead to deficiencies in basic } \\
\text { science knowledge? An empirical case on anatomy }\end{array}$ & $\begin{array}{l}\text { Prince, } 2003 \\
{[88]}\end{array}$ & 129 & 7.2 & $\begin{array}{l}\text { Medical } \\
\text { Education }\end{array}$ & Research & Netherlands \\
\hline 57 & $\begin{array}{l}\text { Using technology to assist in realizing effective learning and } \\
\text { instruction: A principled approach to the use of computers in } \\
\text { collaborative learning }\end{array}$ & $\begin{array}{l}\text { Koschmann, } \\
1994[89]\end{array}$ & 127 & 4.7 & $\begin{array}{l}\text { Journal of the } \\
\text { Learning } \\
\text { Sciences }\end{array}$ & Descriptive & USA \\
\hline 58 & $\begin{array}{l}\text { Roles and responsibilities of the problem based learning tutor in } \\
\text { the undergraduate medical curriculum }\end{array}$ & $\begin{array}{l}\text { Maudsley, } \\
1999[90]\end{array}$ & 126 & 5.7 & $\begin{array}{l}\text { British } \\
\text { Medical } \\
\text { Journal }\end{array}$ & Descriptive & UK \\
\hline
\end{tabular}




\begin{tabular}{|c|c|c|c|c|c|c|c|}
\hline 59 & $\begin{array}{l}\text { Blending problem-based learning with Web technology } \\
\text { positively impacts student learning outcomes in acid-based } \\
\text { physiology }\end{array}$ & $\begin{array}{l}\text { Taradi, } 2005 \\
{[91]}\end{array}$ & 122 & 7.6 & $\begin{array}{l}\text { American } \\
\text { Journal of } \\
\text { Physiology - } \\
\text { Advances in } \\
\text { Physiology } \\
\text { Education }\end{array}$ & Research & Croatia \\
\hline 60 & $\begin{array}{l}\text { Development of self-directed learning behaviors in a partially } \\
\text { teacher-directed problem-based learning curriculum }\end{array}$ & $\begin{array}{l}\text { Blumberg, } \\
1992[92]\end{array}$ & 120 & 4.1 & $\begin{array}{l}\text { Teaching and } \\
\text { Learning in } \\
\text { Medicine }\end{array}$ & Research & Canada \\
\hline 61 & $\begin{array}{l}\text { Undergraduate medical education: comparison of problem-based } \\
\text { learning and conventional teaching }\end{array}$ & $\begin{array}{l}\text { Nandi, } 2000 \\
{[22]}\end{array}$ & 119 & 5.7 & $\begin{array}{l}\text { Hong Kong } \\
\text { Medical } \\
\text { Journal }\end{array}$ & Review & China \\
\hline 62 & $\begin{array}{l}\text { Influence of tutors' subject-matter expertise on student effort and } \\
\text { achievement in problem-based learning }\end{array}$ & $\begin{array}{l}\text { Schmidt, } 1993 \\
\text { [93] }\end{array}$ & 117 & 4.2 & $\begin{array}{l}\text { Academic } \\
\text { Medicine }\end{array}$ & Research & Netherlands \\
\hline 63 & $\begin{array}{l}\text { Problem-based learning in American medical education: An } \\
\text { overview }\end{array}$ & $\begin{array}{l}\text { Donner, } 1993 \\
{[94]}\end{array}$ & 113 & 4.0 & $\begin{array}{l}\text { Bulletin of the } \\
\text { Medical } \\
\text { Library } \\
\text { Association }\end{array}$ & Descriptive & USA \\
\hline 64 & $\begin{array}{l}\text { Changing the attitudes of 'tomorrow's doctors' towards mental } \\
\text { illness and psychiatry: A comparison of two teaching methods }\end{array}$ & $\begin{array}{l}\text { Singh, } 1998 \\
{[95]}\end{array}$ & 111 & 4.8 & $\begin{array}{l}\text { Medical } \\
\text { Education }\end{array}$ & Research & UK \\
\hline 65 & $\begin{array}{l}\text { Long-term outcomes of the new pathway program at Harvard } \\
\text { Medical School: A randomized controlled trial }\end{array}$ & $\begin{array}{l}\text { Peters, } 2000 \\
{[96]}\end{array}$ & 110 & 5.2 & $\begin{array}{l}\text { Academic } \\
\text { Medicine }\end{array}$ & Research & USA \\
\hline 66 & $\begin{array}{l}\text { Problem based learning: Cognitive and metacognitive processes } \\
\text { during problem analysis }\end{array}$ & $\begin{array}{l}\text { De Grave, } 1996 \\
{[97]}\end{array}$ & 110 & 4.4 & $\begin{array}{l}\text { Instructional } \\
\text { Science }\end{array}$ & Research & Netherlands \\
\hline 67 & Problem-based learning: Where are we now? & $\begin{array}{l}\text { Taylor, } 2008 \\
{[47]}\end{array}$ & 109 & 7.8 & $\begin{array}{l}\text { Medical } \\
\text { Teacher }\end{array}$ & Descriptive & UK \\
\hline 68 & $\begin{array}{l}\text { Perceptions of how well graduates are prepared for the role of } \\
\text { pre-registration house officer: A comparison of outcomes from a } \\
\text { traditional and an integrated PBL curriculum }\end{array}$ & $\begin{array}{l}\text { Jones, } 2002 \\
{[98]}\end{array}$ & 108 & 5.7 & $\begin{array}{l}\text { Medical } \\
\text { Education }\end{array}$ & Research & UK \\
\hline
\end{tabular}




\begin{tabular}{|c|c|c|c|c|c|c|c|}
\hline 69 & Problem-based learning: Its rationale and efficacy & $\begin{array}{l}\text { Finucane, } 1998 \\
\text { [99] }\end{array}$ & 108 & 4.7 & $\begin{array}{l}\text { Medical } \\
\text { Journal of } \\
\text { Australia }\end{array}$ & Descriptive & Australia \\
\hline 70 & $\begin{array}{l}\text { Problem-based learning in pre-clinical medical education: } 22 \\
\text { years of outcome research }\end{array}$ & $\begin{array}{l}\text { Hartling, } 2010 \\
{[21]}\end{array}$ & 107 & 9.7 & $\begin{array}{l}\text { Medical } \\
\text { Teacher }\end{array}$ & Review & Canada \\
\hline 71 & $\begin{array}{l}\text { Profiles of effective tutors in problem-based learning: } \\
\text { Scaffolding student learning }\end{array}$ & $\begin{array}{l}\text { De Grave, } 1999 \\
\text { [42] }\end{array}$ & 106 & 4.8 & $\begin{array}{l}\text { Medical } \\
\text { Education }\end{array}$ & Research & Netherlands \\
\hline 72 & The advantages of problem-based curricula & $\begin{array}{l}\text { Dolmans, } 1996 \\
\text { [39] }\end{array}$ & 105 & 4.2 & $\begin{array}{l}\text { Postgraduate } \\
\text { Medical } \\
\text { Journal } \\
\end{array}$ & Descriptive & Netherlands \\
\hline 73 & $\begin{array}{l}\text { Seven principles of effective case design for a problem-based } \\
\text { curriculum }\end{array}$ & $\begin{array}{l}\text { Dolmans, } 1997 \\
{[100]}\end{array}$ & 102 & 4.3 & $\begin{array}{l}\text { Medical } \\
\text { Teacher }\end{array}$ & Descriptive & Netherlands \\
\hline 74 & $\begin{array}{l}\text { PBL in the undergraduate MD program at McMaster University: } \\
\text { Three iterations in three decades }\end{array}$ & $\begin{array}{l}\text { Neville, } 2007 \\
{[101]}\end{array}$ & 100 & 7.1 & $\begin{array}{l}\text { Academic } \\
\text { Medicine }\end{array}$ & Descriptive & Canada \\
\hline 75 & $\begin{array}{l}\text { The attitudes of "tomorrow's doctors" towards mental illness and } \\
\text { psychiatry: Changes during the final undergraduate year }\end{array}$ & $\begin{array}{l}\text { Baxter, } 2001 \\
{[102]}\end{array}$ & 100 & 5.0 & $\begin{array}{l}\text { Medical } \\
\text { Education } \\
\end{array}$ & Research & UK \\
\hline 76 & $\begin{array}{l}\text { Effects of tutors with subject expertise on the problem-based } \\
\text { tutorial process }\end{array}$ & $\begin{array}{l}\text { Silver, } 1991 \\
{[103]}\end{array}$ & 97 & 3.2 & $\begin{array}{l}\text { Academic } \\
\text { Medicine }\end{array}$ & Research & USA \\
\hline 77 & $\begin{array}{l}\text { Problem-based learning outcomes: Ten years of experience at } \\
\text { the University of Missouri-Columbia School of Medicine }\end{array}$ & $\begin{array}{l}\text { Hoffman, } 2006 \\
\text { [104] }\end{array}$ & 96 & 6.4 & $\begin{array}{l}\text { Academic } \\
\text { Medicine }\end{array}$ & Research & USA \\
\hline 78 & $\begin{array}{l}\text { Deep and surface learning in problem-based learning: a review } \\
\text { of the literature }\end{array}$ & $\begin{array}{l}\text { Dolmans, } 2016 \\
{[5]}\end{array}$ & 95 & 19.0 & $\begin{array}{l}\text { Advances in } \\
\text { Health } \\
\text { Sciences } \\
\text { Education }\end{array}$ & Review & Netherlands \\
\hline 79 & $\begin{array}{l}\text { Rethinking the globalisation of problem-based learning: How } \\
\text { culture challenges self-directed learning }\end{array}$ & $\begin{array}{l}\text { Frambach, } \\
2012[105]\end{array}$ & 94 & 10.4 & $\begin{array}{l}\text { Medical } \\
\text { Education }\end{array}$ & Research & Netherlands \\
\hline 80 & $\begin{array}{l}\text { Implementation of problem-based learning in Asian medical } \\
\text { schools and students' perceptions of their experience }\end{array}$ & $\begin{array}{l}\text { Khoo, } 2003 \\
{[106]}\end{array}$ & 94 & 5.2 & $\begin{array}{l}\text { Medical } \\
\text { Education } \\
\end{array}$ & Descriptive & Singapore \\
\hline 81 & $\begin{array}{l}\text { An analysis of peer, self, and tutor assessment in problem-based } \\
\text { learning tutorials }\end{array}$ & $\begin{array}{l}\text { Papinczak, } \\
2007[48]\end{array}$ & 90 & 6.4 & $\begin{array}{l}\text { Medical } \\
\text { Teacher }\end{array}$ & Research & Australia \\
\hline
\end{tabular}




\begin{tabular}{|c|c|c|c|c|c|c|c|}
\hline 82 & $\begin{array}{l}\text { General competencies of problem-based learning (PBL) and } \\
\text { non-PBL graduates }\end{array}$ & $\begin{array}{l}\text { Prince, } 2005 \\
{[107]}\end{array}$ & 88 & 5.5 & $\begin{array}{l}\text { Medical } \\
\text { Education }\end{array}$ & Research & Netherlands \\
\hline 83 & $\begin{array}{l}\text { A conceptual framework to guide the development of self- } \\
\text { directed, lifelong learning in problem-based medical curricula }\end{array}$ & $\begin{array}{l}\text { Miflin, } 2000 \\
{[9]}\end{array}$ & 88 & 4.2 & $\begin{array}{l}\text { Medical } \\
\text { Education }\end{array}$ & Theoretical & Australia \\
\hline 84 & What drives the student in problem-based learning? & $\begin{array}{l}\text { Dolmans, } 1994 \\
{[108]}\end{array}$ & 88 & 3.3 & $\begin{array}{l}\text { Medical } \\
\text { Education }\end{array}$ & Research & Netherlands \\
\hline 85 & $\begin{array}{l}\text { Effects of tutors with case expertise on problem-based learning } \\
\text { issues }\end{array}$ & $\begin{array}{l}\text { Eagle, } 1992 \\
{[109]}\end{array}$ & 88 & 3.0 & $\begin{array}{l}\text { Academic } \\
\text { Medicine }\end{array}$ & Research & Canada \\
\hline 86 & $\begin{array}{l}\text { A comparison of critical thinking in groups of third-year medical } \\
\text { students in text, video, and virtual pbl case modalities }\end{array}$ & $\begin{array}{l}\text { Kamin, } 2003 \\
{[110]}\end{array}$ & 87 & 4.8 & $\begin{array}{l}\text { Academic } \\
\text { Medicine }\end{array}$ & Research & USA \\
\hline 87 & $\begin{array}{l}\text { A comparison of in-class learner engagement across lecture, } \\
\text { problem-based learning, and team learning using the STROBE } \\
\text { classroom observation tool }\end{array}$ & $\begin{array}{l}\text { Kelly, } 2005 \\
{[111]}\end{array}$ & 86 & 5.4 & $\begin{array}{l}\text { Teaching and } \\
\text { Learning in } \\
\text { Medicine }\end{array}$ & Research & USA \\
\hline 88 & Group problems in problem-based learning & $\begin{array}{l}\text { Hendry, } 2003 \\
{[112]}\end{array}$ & 86 & 4.8 & $\begin{array}{l}\text { Medical } \\
\text { Teacher }\end{array}$ & Research & Australia \\
\hline 89 & $\begin{array}{l}\text { The many faces of problem-based learning: A framework for } \\
\text { understanding and comparison }\end{array}$ & $\begin{array}{l}\text { Charlin, } 1998 \\
{[113]}\end{array}$ & 86 & 3.7 & $\begin{array}{l}\text { Medical } \\
\text { Teacher }\end{array}$ & Descriptive & Canada \\
\hline 90 & $\begin{array}{l}\text { Shifting students and faculty to a PBL curriculum: Attitudes } \\
\text { changed and lessons learned }\end{array}$ & $\begin{array}{l}\text { Bernstein, } 1995 \\
{[114]}\end{array}$ & 86 & 3.3 & $\begin{array}{l}\text { Academic } \\
\text { Medicine }\end{array}$ & Research & Canada \\
\hline 91 & $\begin{array}{l}\text { The problem-based learning tutor: Teacher? Facilitator? } \\
\text { Evaluator? }\end{array}$ & $\begin{array}{l}\text { Neville, } 1999 \\
\text { [115] }\end{array}$ & 84 & 3.8 & $\begin{array}{l}\text { Medical } \\
\text { Teacher }\end{array}$ & Review & Canada \\
\hline 92 & Problem-based learning: Measurable outcomes & $\begin{array}{l}\text { Thomas, } 1997 \\
{[116]}\end{array}$ & 84 & 3.5 & $\begin{array}{l}\text { Medical } \\
\text { Education } \\
\end{array}$ & Review & Canada \\
\hline 93 & $\begin{array}{l}\text { Student performances on Step } 1 \text { and Step } 2 \text { of the United States } \\
\text { Medical Licensing Examination following implementation of a } \\
\text { problem-based learning curriculum }\end{array}$ & $\begin{array}{l}\text { Blake, } 2000 \\
{[117]}\end{array}$ & 83 & 4.0 & $\begin{array}{l}\text { Academic } \\
\text { Medicine }\end{array}$ & Research & USA \\
\hline 94 & $\begin{array}{l}\text { Comparison of text and video cases in a postgraduate problem- } \\
\text { based learning format }\end{array}$ & $\begin{array}{l}\text { Balslev, } 2005 \\
{[118]}\end{array}$ & 80 & 5.0 & $\begin{array}{l}\text { Medical } \\
\text { Education }\end{array}$ & Research & Denmark \\
\hline
\end{tabular}




\begin{tabular}{|c|c|c|c|c|c|c|c|}
\hline 95 & $\begin{array}{l}\text { Resolving inconsistencies in tutor expertise research: Does lack } \\
\text { of structure cause students to seek tutor guidance? }\end{array}$ & $\begin{array}{l}\text { Schmidt, } 1994 \\
{[119]}\end{array}$ & 79 & 2.9 & $\begin{array}{l}\text { Academic } \\
\text { Medicine }\end{array}$ & Research & Netherlands \\
\hline 96 & Problem-based learning outcomes: The glass half-full & $\begin{array}{l}\text { Distlehorst, } \\
2005[120]\end{array}$ & 78 & 4.9 & $\begin{array}{l}\text { Academic } \\
\text { Medicine }\end{array}$ & Research & USA \\
\hline 97 & $\begin{array}{l}\text { How can medical students learn in a self-directed way in the } \\
\text { clinical environment? Design-based research. }\end{array}$ & $\begin{array}{l}\text { Dornan, } 2005 \\
{[121]}\end{array}$ & 78 & 4.9 & $\begin{array}{l}\text { Medical } \\
\text { Education }\end{array}$ & Research & UK \\
\hline 98 & Problem effectiveness in a course using problem-based learning & $\begin{array}{l}\text { Dolmans, } 1993 \\
{[122]}\end{array}$ & 77 & 2.8 & $\begin{array}{l}\text { Academic } \\
\text { Medicine }\end{array}$ & Research & Netherlands \\
\hline 99 & $\begin{array}{l}\text { Student-led tutorials in problem-based learning: Educational } \\
\text { outcomes and students' perceptions }\end{array}$ & $\begin{array}{l}\text { Kassab, } 2005 \\
{[123]}\end{array}$ & 76 & 4.8 & $\begin{array}{l}\text { Medical } \\
\text { Teacher }\end{array}$ & Research & Bahrain \\
\hline 100 & $\begin{array}{l}\text { Do junior doctors feel they are prepared for hospital practice? A } \\
\text { study of graduates from traditional and non-traditional medical } \\
\text { schools }\end{array}$ & $\begin{array}{l}\text { Hill, } 1998 \\
{[124]}\end{array}$ & 76 & 3.3 & $\begin{array}{l}\text { Medical } \\
\text { Education }\end{array}$ & Research & Australia \\
\hline
\end{tabular}


Citation count

Overall, all 100 articles had a total of 27,946 citations at the time of the search. The range of citations for the 100 articles was between 76 and 2,997 times cited. The most cited article had 2,997 citations over 15 years with mean citations per year of 199.8 whereas the article ranked 100 had 76 citations over 23 years with mean citations per year of 3.3. Of the 100 articles, the least average citations per year was 2.8 for an article that had 77 citations over 28 years. Publication years

The oldest article was published in 1981 and the most recent article was published in 2016, a span of 35 years. Grouping publication years by decade, 46 articles were published between 1991-2000, followed by 41 articles between 2001-2010, then 10 between 1981-1990, and 3 between 2011-2020. With regard to the number of articles published by year, 2005 had the highest number of publications (9) and 8 articles were published each in 2000 and 1993. There was no correlation between the number of citations for an article and the number of years since it was published $\left(R^{2}=0.013, p=.898\right)$.

Authors and author collaboration

Two hundred and twelve authors contributed to the 100 most cited articles. The most prolific authors who had five or more publications include, Schmidt HG who authored/coauthored 18 articles, followed by Dolmans DHJM with 10 articles, van der Vleuten CPM (9), Barrows HS (7), Norman GR (6) and De Grave WS (5). Thirty-nine articles had 1-2 authors, 48 articles had 3-4 authors, 12 articles had 5-7 authors, and one article had 11 authors. Twenty authors were the sole authors of one article each and 35 authors coauthored two articles or more.

Using VOSviewer, we explored the collaborative relationships between authors. VOSviewer uses the visualization of similarities mapping technique to construct maps and 
display items based on their weight [38]. Figure 1 shows a network visualization of the largest set of author collaboration. The size of the node denotes the number of articles published by each author and the lines between nodes represent collaboration links. The co-authorship network showed that Schmidt HG had the strongest collaboration (32 total link strength) followed by van der Vleuten CPM (30), and Dolmans DHJM (25).

Figure 1. Network visualization of the largest set of author collaboration

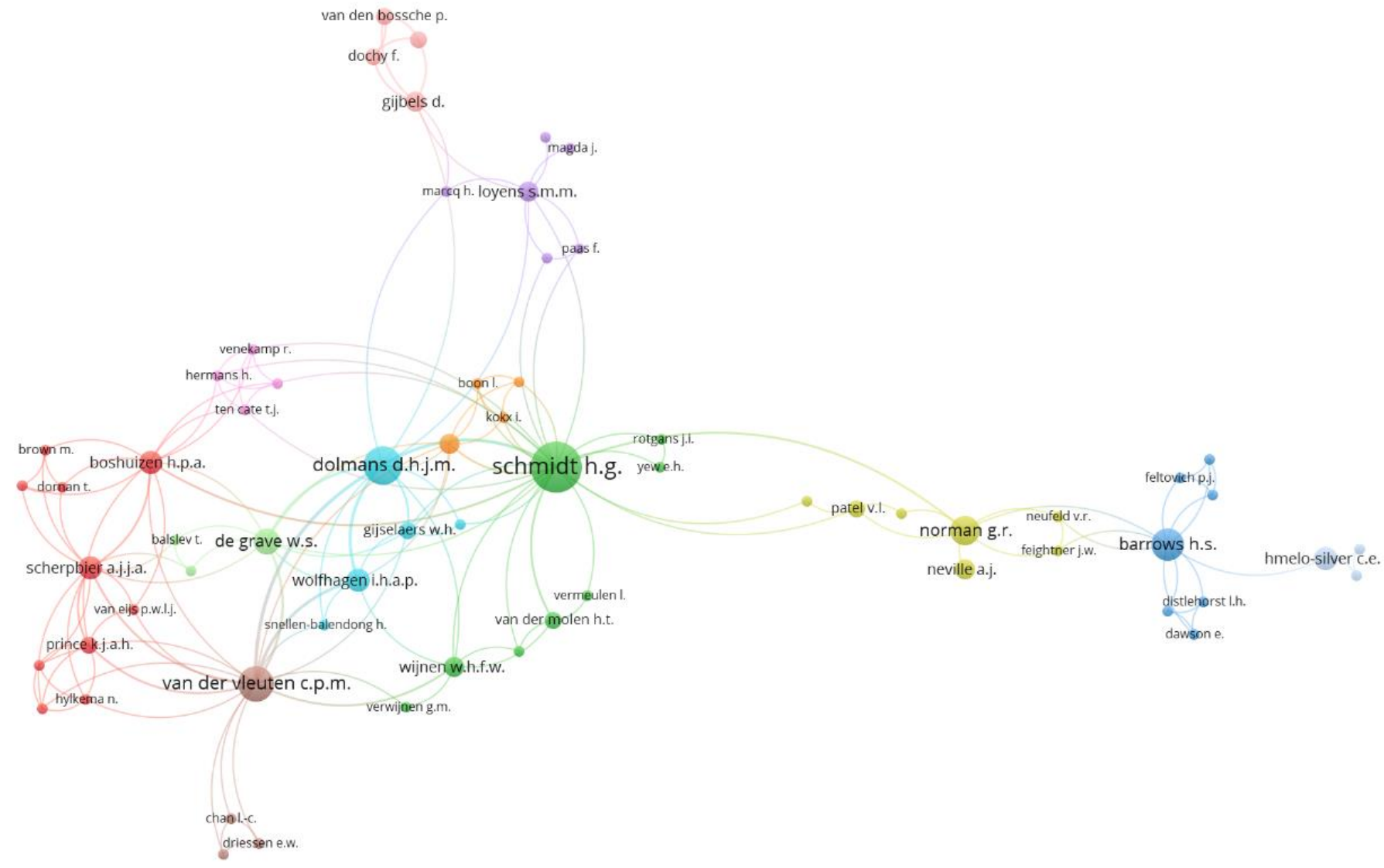

Country of first author at publication time

The 212 authors were from 13 countries and the first authors were from 11 countries at the time of publication. The Netherlands had the highest number of first authors $(n=30,30 \%)$, followed by Canada $(n=23,23 \%)$, US $(n=18,18 \%)$, UK $(n=15,15 \%)$, Australia $(n=6,6 \%)$, two each $(2 \%)$ from Germany and Singapore, and 1 each (1\%) from Bahrain, China, Croatia, and 
Denmark. With regard to collaboration between countries (see Figure 2), Netherlands had the strongest collaboration network (15 total link strength) followed by US (5), and Canada (5). Three of the 13 countries were not connected in the collaboration network.

Figure 2. Network visualization of collaboration between countries

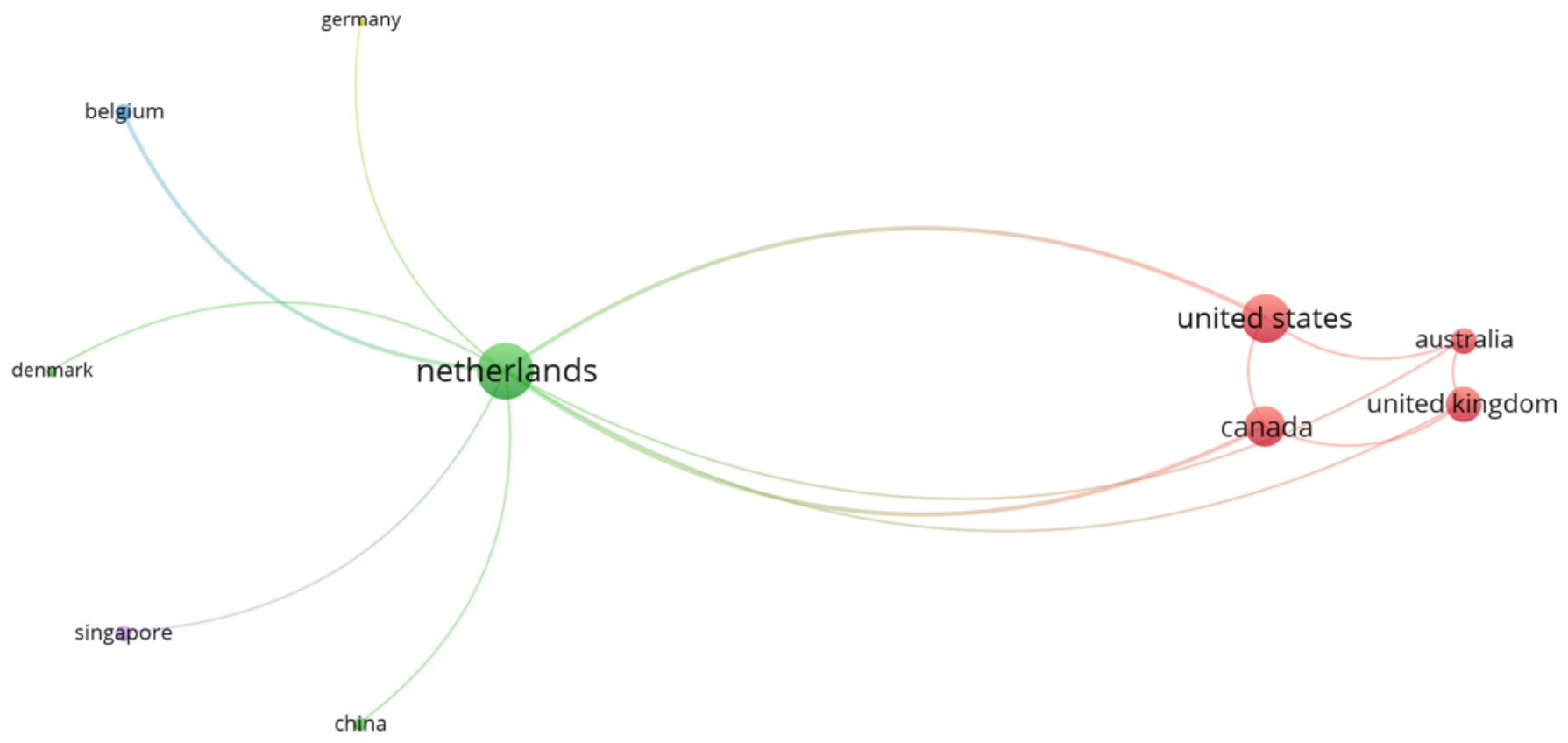

Journals

The 100 most cited articles were published in 23 journals (Table 2). The journals are listed in descending order by number of articles and journals with the same absolute number of articles are ordered by the h-index, which is a journal's number of articles $(\mathrm{h})$ that have received at least $\mathrm{h}$ citations. The top three journals with the highest frequency of articles cited were, Medical Education $(n=32,32 \%)$, Academic Medicine $(n=25,25 \%)$, and Medical Teacher $(n=$ $11,11 \%)$. Seventeen (74\%) of the 23 journals were non-medical education journals and included journals in general education, educational psychology, as well as general and critical care medicine. Twenty-six (26\%) of the most-cited 100 articles were published in the 17 non-medical education journals. 
The journal CiteScore, which measures the citation impact of the journal, shows that Journal of the American Medical Association (JAMA) had the highest CiteScore (24.8 in 2020) followed by Review of Educational Research (18.4) and Educational Psychologist (14.9). For the h-index, JAMA had the highest h-index (680) followed by British Medical Journal (429) and Critical Care Medicine (271). Medical Education, Academic Medicine, and Medical Teacher had CiteScores of 5.3, 5.4, and 4.5, and h-indices of 138, 152, and 109, respectively.

Table 2. Journals in which the 100 most cited articles on PBL in medical education were published

\begin{tabular}{|c|c|c|c|c|}
\hline Rank & Journal & $\begin{array}{c}\text { Number of } \\
\text { articles }\end{array}$ & $\begin{array}{c}\text { CiteScore } \\
(2020)\end{array}$ & h-index \\
\hline 1 & Medical Education & 32 & 5.3 & 138 \\
\hline 2 & Academic Medicine & 25 & 5.4 & 152 \\
\hline 3 & Medical Teacher & 11 & 4.5 & 109 \\
\hline 4 & British Medical Journal & 4 & 6.5 & 429 \\
\hline 5 & Educational Psychologist & 4 & 14.9 & 126 \\
\hline 6 & Advances in Health Sciences Education & 3 & 4.9 & 64 \\
\hline 7 & Canadian Medical Association Journal & 2 & 4.5 & 186 \\
\hline 8 & Educational Psychology Review & 2 & 13.3 & 111 \\
\hline 9 & Journal of the Learning Sciences & 2 & 9.7 & 92 \\
\hline 10 & Teaching and Learning in Medicine & 2 & 3.0 & 48 \\
\hline 11 & Journal of the American Medical Association & 1 & 24.8 & 680 \\
\hline 12 & Critical Care Medicine & 1 & 12.7 & 271 \\
\hline 13 & Review of Educational Research & 1 & 18.4 & 153 \\
\hline 14 & Medical Journal of Australia & 1 & 4.8 & 131 \\
\hline 15 & Learning and Instruction & 1 & 8.2 & 116 \\
\hline 16 & Postgraduate Medical Journal & 1 & 3.3 & 99 \\
\hline 17 & Cognition and Instruction & 1 & 5.4 & 81 \\
\hline 18 & Instructional Science & 1 & 4.8 & 75 \\
\hline 19 & $\begin{array}{l}\text { American Journal of Physiology - Advances } \\
\text { in Physiology Education }\end{array}$ & 1 & 2.4 & 60 \\
\hline 20 & $\begin{array}{l}\text { Bulletin of the Medical Library Association } \\
\text { (now Journal of the Medical Library } \\
\text { Association) }\end{array}$ & 1 & 3.0 & 58 \\
\hline 21 & Hong Kong Medical Journal & 1 & 1.6 & 46 \\
\hline 22 & New Directions for Teaching and Learning & 1 & 0.8 & 46 \\
\hline 23 & Medical Principles and Practice & 1 & 2.7 & 45 \\
\hline
\end{tabular}

Types of articles and foci

We categorized articles into four types as shown in Table 1: descriptive, research, review, and theoretical. Based on our definitions of the categories, almost half of the articles were 
research $(n=46,46 \%)$, almost one third were descriptive $(n=31,31 \%)$, and the rest were review articles $(n=19,19 \%)$, and 4 theoretical papers $(4 \%)$. For the research articles, 36 studies employed quantitative methods, seven used mixed-methods, and three were qualitative studies. The review articles included 11 narrative reviews, six meta-analyses, and two systematic reviews.

Table 3 further classifies the types of articles by their focal areas. These focal areas include nine categories that emerged from full paper review. The focal areas were as follows with examples across article types where applicable: learning outcomes $(n=42,42 \%$, e.g., [20,39]; learning process $(n=17,17 \%$, e.g., $[11,40]$; curriculum $(n=16,16 \%$, e.g., $[13,41]$; tutor $(n=12,12 \%$, e.g., $[42,43]$; theoretical $(n=4,4 \%$, e.g., [7,9]; commentary $(n=3,3 \%$, e.g., [44,45]; guide ( $n=3,3 \%$, e.g., [46,47]; assessment $(n=2,2 \%,[48,49]$; and student well-being $(n=1,1 \%,[50]$. 
Table 3. The 100 Most Cited Articles on Problem-based Learning in Medical Education by Article Type and Focus

\begin{tabular}{|c|c|c|c|c|c|}
\hline Article Focus & Descriptive & Research & Review & Theoretical & Total (\%) \\
\hline Learning Outcomes & $\begin{array}{l}\text { Colliver, 2000; Berkson, } \\
\text { 1993; Dolmans, } 1996\end{array}$ & $\begin{array}{l}\text { Steadman, 2006; Newble, 1986; } \\
\text { Shin, 1993; Schmidt, 1996; } \\
\text { Schmidt, 2006; Coles, 1985; } \\
\text { Neufeld, 1981; Hmelo, 1998; } \\
\text { Patel, 1991; Antephol, 1999; } \\
\text { McParland, 2004; Woltering, } \\
\text { 2009; Prince, 2003; Taradi, 2005; } \\
\text { Blumberg, 1992; Singh, 1998; } \\
\text { Peters, 2000; De Grave, 1996; } \\
\text { Jones, 2002; Baxter, 2001; } \\
\text { Hoffman, 2006; Prince, 2005; } \\
\text { Kamin, 2003; Blake, 2000; } \\
\text { Distlehorst, 2005; Hill, 1998 }\end{array}$ & $\begin{array}{l}\text { Albanese, 1993; Vernon, } \\
\text { 1993; Dochy, 2003; Gijbels, } \\
\text { 2005; Koh, 2008; Neville, } \\
\text { 2008; Smits, 2002; Schmidt, } \\
\text { 2009; Schmidt, 1987; Nandi, } \\
\text { 2000; Hartling, 2010; } \\
\text { Dolmans, 2016; Thomas, } \\
1997\end{array}$ & & $42(42 \%)$ \\
\hline Curriculum & $\begin{array}{l}\text { Barrows, 1996; Wood, 2003; } \\
\text { Spencer, 1999; Walton, 1989; } \\
\text { Barrows, 1983; Donner, } \\
\text { 1993; Finucane, 1998; } \\
\text { Dolmans, 1997; Neville, } \\
\text { 2007; Khoo, 2003; Charlin, } \\
\text { 1998 }\end{array}$ & $\begin{array}{l}\text { Srinivasan, 2007; Kaufman, } \\
\text { 1989; Bernstein, 1995; Balslev, } \\
2005\end{array}$ & Maudsley, 1999 & & $16(16 \%)$ \\
\hline Learning Process & $\begin{array}{l}\text { Schmidt, 1983; Dolmans, } \\
\text { 2005; Schmidt, 2011; } \\
\text { Loyens, 2008; Maudsley, } \\
\text { 2000; Mandin, 1997; } \\
\text { Koschmann, } 1994\end{array}$ & $\begin{array}{l}\text { Frambach, 2012; Dolmans, 1994; } \\
\text { Kelly, 2005; Hendry, 2003; } \\
\text { Dornan, 2005; Dolmans, } 1993\end{array}$ & $\begin{array}{l}\text { Norman, 1992; Hmelo-Silver, } \\
\text { 2004; Norman, 1988; } \\
\text { Dolmans, } 2006\end{array}$ & & $17(17 \%)$ \\
\hline Tutor & $\begin{array}{l}\text { Dolmans, 2002; Dolmans, } \\
\text { 2001; Maudsley, } 1999\end{array}$ & $\begin{array}{l}\text { Hmelo-Silver, 2008; Schmidt, } \\
\text { 1995; Schmidt, 1993; De Grave, } \\
\text { 1999; Silver, 1991; Eagle, 1992; } \\
\text { Schmidt, 1994; Kassab, } 2005\end{array}$ & Neville, 1999 & & $12(12 \%)$ \\
\hline Theoretical & & & & $\begin{array}{l}\text { Kirschner, 2006; Schmidt, } \\
\text { 1993; Albanese, 2000; } \\
\text { Miflin, } 2000\end{array}$ & $4(4 \%)$ \\
\hline Commentary & $\begin{array}{l}\text { Hmelo-Silver, 2007; } \\
\text { Norman, 2000; Schmidt, } \\
2007\end{array}$ & & & & $3(3 \%)$ \\
\hline
\end{tabular}




\begin{tabular}{|c|c|c|c|c|c|}
\hline Guide & $\begin{array}{l}\text { Barrows, 1986; Davis, 1999; } \\
\text { Taylor, } 2008\end{array}$ & & & & $3(3 \%)$ \\
\hline Assessment & Van Der Vleuten, 1996 & Papinczak, 2007 & & & $2(2 \%)$ \\
\hline Student well-being & & Moffat, 2004 & & & $1(1 \%)$ \\
\hline Total $(\%)$ & $31(31 \%)$ & $46(46 \%)$ & $19(19 \%)$ & $4(4 \%)$ & $100(100 \%)$ \\
\hline
\end{tabular}




\section{Discussion}

This bibliometric analysis sought to analyze the characteristics of the 100 most cited articles on PBL in medical education. The number of citations ranged from 76 to 2,997 times cited with 27,946 total citations. The articles were published from 1981 through 2016, as articles published in the last five years may not have enough time to accrue citations to determine their impact [51]. The most prolific decade was 1991-2000 followed by 2001-2010. There was a peak in the number of highly cited articles in 2005 and the number seemed to taper off in subsequent years. However, an article that was published in 2016 ranked higher than articles that were published in the early 1990s, implying that the year of publication did not have much effect on an article's number of citations. This is supported by the no correlation found between the number of citations for an article and the number of years since it was published. This result is consistent with previous findings [29,32].

It is fitting that over $60 \%$ of the most cited articles were published in Medical Education, Academic Medicine, and Medical Teacher since these are the top three most productive medical education journals as evidenced by their high CiteScores among medical education journals. Of the 23 journals with the most cited articles, the top 3 journals with the highest CiteScores were non-medical education journals, namely Journal of the American Medical Association, which had 1 article, Review of Educational Research (1), and Educational Psychologist (4). Similar to previous work, there was no correlation between the total number of citations and the journal CiteScore [32].

With regard to countries, 13 countries contributed to the most cited articles. This speaks to the fact that PBL had garnered global attention as an innovative instructional approach and more medical schools were adopting it. At present, the most recent curriculum inventory data 
reported to the AAMC shows that 68 out of 153 (44\%) medical schools use PBL in their curriculum [3]. Further, PBL as a percentage of all instructional methods was $1.05 \%$ in 2013 , the first year for which data was reported, and 2.09\% in 2020 [52]. It is unclear, however, whether these schools implement the core model or a different model of PBL [13,53]. It is possible that some medical schools confound PBL with case-based instruction, which also uses patient cases. Low fidelity of implementation is likely the reason for the problems encountered in the classroom [16]. Hence, evidence of how PBL is currently being implemented is needed.

Authors in Netherlands, US, Canada, and UK contributed more than $80 \%$ of the articles. We identified six prolific authors who had five or more publications and authored a total of 55 articles. Co-authorship analysis indicated strong collaborations between countries, with the strongest collaboration network between authors in Netherlands, US, and Canada. This might be attributed to the fact that Canada was the pioneer of PBL while Netherlands and US were among the early adopters, [54] thus, facilitating collaborations between these three countries. It is not surprising that the majority of the articles were empirical studies focused on the effectiveness of PBL and student learning outcomes. Most of the highly cited research studies were published through the mid-2000s, which is consistent with Hung et al.'s [24] first wave of research that sought to investigate whether PBL works. The debate on the effectiveness of PBL over conventional instructional methods has been ongoing and more research is needed on the learning outcomes from the implementation of the different PBL models.

One major observation of this bibliometric analysis was the paucity of studies that used qualitative methods that were highly cited. A possible explanation is that robust qualitative studies require ample time to conduct. Another observation was the insufficient number of longitudinal studies examining the long-term impact of PBL. Most studies on the effects of PBL 
have been conducted in undergraduate medical education. Koh et al., [18] found only 13 studies that assessed physician competencies of first-year residents who were taught using PBL in medical school. Further, it appears that the number of such studies looking at physician competencies well beyond residency is limited. While it might be difficult to conduct follow-up studies with graduates when they are well into practice, it is important to ascertain the long-term impact of PBL in order to contribute to the ongoing debate about its effectiveness.

\section{Limitations}

Our study has a number of limitations. First, we only used a single database for our search and it is possible that another database such as Web of Science could yield different numbers of citations. We sought advisement from a research librarian who affirmed that Scopus was the most comprehensive peer-reviewed research database available at our institution. Second, our study only included English articles, however, the most cited non-English article on PBL in medical education had 27 citations and would not have made the list of top 100 most cited articles. Third, we did not exclude self-citations, which may introduce intrinsic bias. Kacem and colleagues [55] argued, however, that excluding self-citations distorts the record of progress and discovery, and the authors proposed a way to report all citation data while accounting for self-citations. Lastly, article selection for inclusion is fairly subjective and other researchers may have different perceptions of the 100 top-cited articles. For example, some articles had the words "problem-based" in the title but upon further review, we found that the articles' focus was on another aspect of medical education, for instance, 'the importance of vertical integration in a problem-based curriculum.' We reviewed these types of articles thoroughly and discussed why they did not meet inclusion criteria since their central topic was not problem-based learning as an instructional approach in medical education. 


\section{Conclusion}

In summary, the purpose of this study was to analyze the bibliometric characteristics of the 100 most cited articles on PBL in medical education. The articles were published in 23 journals by 212 authors from 13 countries between 1981 and 2016, with 1991-2010 being the most prolific period. Over three-fifths of the articles were published in Medical Education, Academic Medicine, and Medical Teacher and research articles represented almost a half of the publications with most using quantitative methods. PBL in medical education is likely to remain an active topic of research as the debate on its effectiveness continues. Our findings are useful for researchers to understand the current state of knowledge in the field as well as identify landmark papers that have made significant contributions to PBL research in medical education. This article also provides a resource on PBL for researchers new to problem-based learning research in medical education. Our study highlights gaps in a dearth of qualitative studies, longitudinal research on the long-term impact of PBL, and more studies are needed on how PBL is actually being implemented as well as the student learning outcomes.

\section{Acknowledgment}

N/A

\section{Declaration of interest}

The authors declare no conflicts of interest. 


\section{References}

1. Jonas HS, Etzel SI, Barzansky B. Educational programs in US medical schools. JAMA. 1992;268:1083-90.

2. Kinkade S. A snapshot of the status of problem-based learning in U. S. medical schools, 2003-04. Acad Med. 2005;80:300-1.

3. Association of American Medical Colleges. Instructional formats used in the curriculum. 2021. https://www.aamc.org/data-reports/curriculum-reports/interactive-data/instructionalformats-used-curriculum. Accessed 17 Jul 2021.

4. Albanese M. Problem-based learning: why curricula are likely to show little effect on knowledge and clinical skills. Med Educ. 2000;34:729-38.

5. Dolmans DHJM, Loyens SMM, Marcq H, Gijbels D. Deep and surface learning in problembased learning: a review of the literature. Adv Health Sci Educ. 2016 Dec;21:1087-112.

6. Gijbels D, Dochy F, van den Bossche P, Segers M. Effects of problem-based learning: a meta-analysis from the angle of assessment. Rev Educ Res. 2005;75:27-61.

7. Kirschner PA, Sweller J, Clark RE. Why minimal guidance during instruction does not work: an analysis of the failure of constructivist, discovery, problem-based, experiential, and inquiry-based teaching. Educ Psychol. 2006;41:75-86.

8. Loyens SMM, Magda J, Rikers RMJP. Self-Directed Learning in Problem-Based Learning and its Relationships with Self-Regulated Learning. Educ Psychol Rev. 2008 Dec $1 ; 20(4): 411-27$.

9. Miflin BM, Campbell CB, Price DA. A conceptual framework to guide the development of self-directed, lifelong learning in problem-based medical curricula. Med Educ. 2000;34:299_ 306. 
10. Norman GR, Schmidt HG. The psychological basis of problem-based learning: a review of the evidence. Acad Med. 1992;67:557-65.

11. Schmidt HG. Problem-based learning: rationale and description. Med Educ. 1983;17:11-6.

12. Schmidt HG. Foundations of problem-based learning: some explanatory notes. Med Educ. $1993 ; 27: 422-32$.

13. Barrows HS. Problem-based learning in medicine and beyond: a brief overview. New Dir Teach Learn. 1996;68:3-12.

14. Neufeld VR, Barrows HS. The "McMaster Philosophy": an approach to medical education. Acad Med. 1974;49:1040-50.

15. Barrows HS, Tamblyn RM. Problem-Based Learning: An Approach to Medical Education. Springer Publishing Company; 1980.

16. Dolmans DHJM, Grave WD, Wolfhagen IHAP, van der Vleuten CPM. Problem-based learning: future challenges for educational practice and research. Med Educ. 2005;39:73241.

17. Dochy F, Segers M, van den Bossche P, Gijbels D. Effects of problem-based learning: a meta-analysis. Learn Instr. 2003;13:533-68.

18. Koh GC-H, Khoo HE, Wong ML, Koh D. The effects of problem-based learning during medical school on physician competency: a systematic review. Can Med Assoc J. $2008 ; 178: 34-41$.

19. Vernon DT, Blake RL. Does problem-based learning work? a meta-analysis of evaluative research. Acad Med. 1993;68:550-63.

20. Albanese MA, Mitchell S. Problem-based learning: a review of literature on its outcomes and implementation issues. Acad Med. 1993;68:52-81. 
21. Hartling L, Spooner C, Tjosvold L, Oswald A. Problem-based learning in pre-clinical medical education: 22 years of outcome research. Med Teach. 2010;32:28-35.

22. Nandi PL, Chan JN, Chan CP, Chan P, Chan LP. Undergraduate medical education: comparison of problem-based learning and conventional teaching. Hong Kong Med J. 2000;6:301-6.

23. Smits PBA. Problem based learning in continuing medical education: a review of controlled evaluation studies. BMJ. 2002;324:153-6.

24. Hung W, Dolmans DHJM, van Merriënboer JJG. A review to identify key perspectives in PBL meta-analyses and reviews: trends, gaps and future research directions. Adv Health Sci Educ. 2019;24:943-57.

25. Broadus RN. Toward a definition of "bibliometrics." Scientometrics. 1987;12:373-9.

26. Okubo Y. Bibliometric indicators and analysis of research systems: methods and examples. Paris: OECD Publishing; 1997. OECD Science, Technology and Industry Working Papers, No. $1997 / 01$.

27. Sampson M, Horsley T, Doja A. A Bibliometric analysis of evaluative medical education studies: characteristics and indexing accuracy. Acad Med. 2013;88:421-7.

28. Azer SA. The top-cited articles in medical education: a bibliometric analysis. Acad Med. 2015;90:1147-61.

29. Azer SA, Azer S. Top-cited articles in medical professionalism: a bibliometric analysis versus altmetric scores. BMJ Open. 2019 ;9:e029433.

30. Young M, St-Onge C, Xiao J, Vachon Lachiver E, Torabi N. Characterizing the literature on validity and assessment in medical education: a bibliometric study. Perspect Med Educ. 2018 Jun;7:182-91. 
31. Maggio LA, Costello JA, Norton C, Driessen EW, Artino Jr AR. Knowledge syntheses in medical education: a bibliometric analysis. Perspect Med Educ. 2021;10:79-87.

32. Azer SA. Top-cited articles in problem-based learning: a bibliometric analysis and quality of evidence assessment. J Dent Educ. 2017;81:458-78.

33. Donthu N, Kumar S, Mukherjee D, Pandey N, Lim WM. How to conduct a bibliometric analysis: an overview and guidelines. J Bus Res. 2021;133:285-96.

34. Grant MJ, Booth A. A typology of reviews: an analysis of 14 review types and associated methodologies. Health Inf Libr J. 2009;26:91-108.

35. Landis JR, Koch GG. The measurement of observer agreement for categorical data. Biometrics. 1977;33:159-74.

36. IBM Corp. IBM SPSS statistics for Windows, version 27.0. Armonk, NY: IBM Corp; 2019.

37. Centre for Science and Technology Studies, Leiden University. VOSviewer for Windows, version 1.6.17. Leiden, Netherlands: Centre for Science and Technology Studies, Leiden University; 2021.

38. van Eck NJ, Waltman L. Software survey: VOSviewer, a computer program for bibliometric mapping. Scientometrics. 2010;84:523-38.

39. Dolmans D, Schmidt H. The advantages of problem-based curricula. Postgrad Med J. 1996;72:535-8.

40. Hmelo-Silver Cindy E. Problem-based learning: what and how do students learn? Educ Psychol Rev. 2004;16:235-66.

41. Maudsley G. Do we all mean the same thing by "problem-based learning"? a review of the concepts and a formulation of the ground rules. Acad Med. 1999;74:178-85. 
42. De Grave WS, Dolmans DHJ, van der Vleuten CPM. Profiles of effective tutors in problembased learning: scaffolding student learning. Med Educ. 1999;33:901-6.

43. Dolmans DHJM, Gijselaers WH, Moust JHC, Grave WS de, Wolfhagen IHAP, van der Vleuten CPM. Trends in research on the tutor in problem-based learning: conclusions and implications for educational practice and research. Med Teach. 2002;24:173-80.

44. Hmelo-Silver CE, Duncan RG, Chinn CA. Scaffolding and achievement in problem-based and inquiry learning: a response to Kirschner, Sweller, and Clark (2006). Educ Psychol. 2007;42:99-107.

45. Norman GR, Schmidt HG. Effectiveness of problem-based learning curricula: theory, practice and paper darts. Med Educ. 2000;34:721-8.

46. Davis MH, Harden RM. AMEE Medical Education Guide No. 15: Problem-based learning: a practical guide. Med Teach. 1999 Jan;21:130-40.

47. Taylor TRDD, Miflin B. Problem-based learning: Where are we now? Med Teach. 2008;30:742-63.

48. Papinczak T, Young L, Groves M, Haynes M. An analysis of peer, self, and tutor assessment in problem-based learning tutorials. Med Teach. 2007;29:e122-32.

49. van der Vleuten CPM, Verwijnen GM, Wijnen WHFW. Fifteen years of experience with progress testing in a problem-based learning curriculum. Med Teach. 1996;18:103-9.

50. Moffat KJ, McConnachie A, Ross S, Morrison JM. First year medical student stress and coping in a problem-based learning medical curriculum. Med Educ. 2004;38:482-91.

51. Ioannidis JPA, Fanelli D, Dunne DD, Goodman SN. Meta-research: evaluation and improvement of research methods and practices. PLoS Biol. 2015;13:e1002264. 
52. Association of American Medical Colleges. Instructional methods used by US and Canadian medical schools. 2021. https://www.aamc.org/data-reports/curriculum-reports/interactivedata/instructional-methods-used-us-and-canadian-medical-schools. Accessed 17 July 2021.

53. Barrows HS. A taxonomy of problem-based learning methods. Med Educ. 1986;20:481-6.

54. Hillen H, Scherpbier A, Wijnen W. History of problem-based learning in medical education. In: van Berkel H, Scherpbier A, Hillen H, van der Vleuten C, editors. Lessons from problembased learning. Oxford: Oxford University Press; 2010. p. 5-11.

55. Kacem A, Flatt JW, Mayr P. Tracking self-citations in academic publishing. Scientometrics. 2020;123:1157-65.

56. Colliver JA. Effectiveness of problem-based learning curricula: research and theory. Acad Med. 2000;75:259-66.

57. Wood DF. Problem based learning. BMJ. 2003;326:328-30.

58. Berkson L. Problem-based learning: have the expectations been met? Acad Med. 1993;68:S79.

59. Schmidt HG, Rotgans JI, Yew EH. The process of problem-based learning: what works and why. Med Educ. 2011;45:792-806.

60. Steadman RH, Coates WC, Huang YM, et al. Simulation-based training is superior to problem-based learning for the acquisition of critical assessment and management skills. Crit Care Med. 2006;34:151-7.

61. Spencer JA, Jordan RK. Learner centred approaches in medical education. BMJ. $1999 ; 318: 1280-3$.

62. Walton HJ, Matthews MB. Essentials of problem-based learning. Med Educ. 1989;23:54258. 
63. Maudsley G, Strivens J. Promoting professional knowledge, experiential learning and critical thinking for medical students. Med Educ. 2000;34:535-44.

64. Hmelo-Silver CE, Barrows HS. Facilitating collaborative knowledge building. Cogn Instr. 2008;26:48-94.

65. Srinivasan M, Wilkes M, Stevenson F, Nguyen T, Slavin S. Comparing problem-based learning with case-based learning: effects of a major curricular shift at two institutions. Acad Med. 2007;82:74-82.

66. Neville AJ. Problem-based learning and medical education forty years on. Med Princ Pract. 2009;18:1-9.

67. Newble DI, Clarke RM. The approaches to learning of students in a traditional and in an innovative problem-based medical school. Med Educ. 1986;20:267-73.

68. Schmidt HG, Moust JH. What makes a tutor effective? a structural-equations modeling approach to learning in problem-based curricula. Acad Med. 1995 Aug;70:708-14.

69. Kaufman A, Mennin S, Waterman R, et al. The New Mexico experiment: educational innovation and institutional change. Acad Med. 1989;64:285-94.

70. Schmidt HG, Loyens SMM, van Gog T, Paas F. Problem-based learning is compatible with human cognitive architecture: commentary on Kirschner, Sweller, and Clark (2006). Educ Psychol. 2007;42:91-7.

71. Shin JH, Haynes RB, Johnston ME. Effect of problem-based, self-directed undergraduate education on life-long learning. CMAJ. 1993;148:969-76.

72. Norman GR. Problem-solving skills, solving problems and problem-based learning. Med Educ. 1988;22:279-86. 
73. Schmidt HG, van der Molen HT, Te Winkel WWR, Wijnen WHFW. Constructivist, problem-based learning does work: a meta-analysis of curricular comparisons involving a single medical school. Educ Psychol. 2009;44:227-49.

74. Schmidt HG, Dauphinee WD, Patel VL. Comparing the effects of problem-based and conventional curricula in an international sample. J Med Educ. 1987;62:305-15.

75. Schmidt HG, Machiels-Bongaerts M, Hermans H, ten Cate TJ, Venekamp R, Boshuizen HP. The development of diagnostic competence: comparison of a problem-based, an integrated, and a conventional medical curriculum. Acad Med. 1996;71:658-64.

76. Schmidt HG, Vermeulen L, van der Molen HT. Longterm effects of problem-based learning: a comparison of competencies acquired by graduates of a problem-based and a conventional medical school. Med Educ. 2006;40:562-7.

77. Coles CR. Differences between conventional and problem-based curricula in their students' approaches to studying. Med Educ. 1985;19:308-9.

78. Neufeld VR, Norman GR, Feightner JW, Barrows HS. Clinical problem-solving by medical students: a cross-sectional and longitudinal analysis. Med Educ. 1981;15:315-22.

79. Barrows HS. Problem-Based, self-directed learning. JAMA. 1983;250:3077-80.

80. Hmelo CE. Problem-based learning: effects on the early acquisition of cognitive skill in medicine. J Learn Sci. 1998;7:173-208.

81. Patel VL, Groen GJ, Norman GR. Effects of conventional and problem-based medical curricula on problem solving. Acad Med. 1991;66:380-9.

82. Antepohl W, Herzig S. Problem-based learning versus lecture-based learning in a course of basic pharmacology: a controlled, randomized study. Med Educ. 1999;33:106-13. 
83. McParland M, Noble LM, Livingston G. The effectiveness of problem-based learning compared to traditional teaching in undergraduate psychiatry. Med Educ. 2004;38:859-67.

84. Woltering V, Herrler A, Spitzer K, Spreckelsen C. Blended learning positively affects students' satisfaction and the role of the tutor in the problem-based learning process: results of a mixed-method evaluation. Adv Health Sci Educ. 2009;14:725.

85. Dolmans DHJM, Schmidt HG. What do we know about cognitive and motivational effects of small group tutorials in problem-based learning? Adv Health Sci Educ Theory Pract. 2006;11:321-36.

86. Dolmans DH, Wolfhagen IH, van der Vleuten CP, Wijnen WH. Solving problems with group work in problem-based learning: hold on to the philosophy. Med Educ. 2001;35:8849.

87. Mandin H, Jones A, Woloschuk W, Harasym P. Helping students learn to think like experts when solving clinical problems. Acad Med. 1997;72:173-9.

88. Prince KJAH, van Mameren H, Hylkema N, Drukker J, Scherpbier AJJA, van der Vleuten CPM. Does problem-based learning lead to deficiencies in basic science knowledge? an empirical case on anatomy. Med Educ. 2003;37:15-21.

89. Koschmann TD, Myers AC, Feltovich PJ, Barrows HS. Using technology to assist in realizing effective learning and instruction: a principled approach to the use of computers in collaborative learning. J Learn Sci. 1993;3:227-64.

90. Maudsley G. Roles and responsibilities of the problem based learning tutor in the undergraduate medical curriculum. BMJ. 1999;318:657-61. 
91. Taradi SK, Taradi M, Radić K, Pokrajac N. Blending problem-based learning with web technology positively impacts student learning outcomes in acid-base physiology. Adv Physiol Educ. 2005 Mar;29:35-9.

92. Blumberg P, Michael JA. Development of self-directed learning behaviors in a partially teacher-directed problem-based learning curriculum. Teach Learn Med. 1992;4:3-8.

93. Schmidt HG, van der Arend A, Moust JH, Kokx I, Boon L. Influence of tutors' subjectmatter expertise on student effort and achievement in problem-based learning. Acad Med. 1993;68:784-91.

94. Donner RS, Bickley H. Problem-based learning in American medical education: an overview. Bull Med Libr Assoc. 1993;81:294-8.

95. Singh SP, Baxter H, Standen P, Duggan C. Changing the attitudes of "tomorrow's doctors" towards mental illness and psychiatry: a comparison of two teaching methods. Med Educ. 1998;32:115-20.

96. Peters AS, Greenberger-Rosovsky R, Crowder C, Block SD, Moore GT. Long-term outcomes of the New Pathway Program at Harvard Medical School: a randomized controlled trial. Acad Med. 2000;75:470-9.

97. De Grave WS, Boshuizen HPA, Schmidt HG. Problem based learning: cognitive and metacognitive processes during problem analysis. Instr Sci. 1996;24:321-41.

98. Jones A, McArdle PJ, O’Neill PA. Perceptions of how well graduates are prepared for the role of pre-registration house officer: a comparison of outcomes from a traditional and an integrated PBL curriculum. Med Educ. 2002;36:16-25.

99. Finucane PM, Johnson SM, Prideaux DJ. Problem-based learning: its rationale and efficacy. Med J Aust. 1998;168:445-8. 
100. Dolmans D, Snellen-Balendong H, Wolfhagen I, van der Vleuten C. Seven principles of effective case design for a problem-based curriculum. Med Teach. 1997;19:185-9.

101. Neville AJ, Norman GR. PBL in the undergraduate MD program at McMaster University: three iterations in three decades. Acad Med. 2007;82:370-4.

102. Baxter H, Singh SP, Standen P, Duggan C. The attitudes of "tomorrow's doctors" towards mental illness and psychiatry: changes during the final undergraduate year. Med Educ. 2001;35:381-3.

103. Silver M, Wilkerson LA. Effects of tutors with subject expertise on the problem-based tutorial process. Acad Med. 1991;66:298-300.

104. Hoffman K, Hosokawa M, Blake R, Headrick L, Johnson G. Problem-based learning outcomes: ten years of experience at the University of Missouri-Columbia School of Medicine. Acad Med. 2006;81:617-25.

105. Frambach JM, Driessen EW, Chan L-C, van der Vleuten CPM. Rethinking the globalisation of problem-based learning: how culture challenges self-directed learning. Med Educ. 2012;46:738-47.

106. Khoo HE. Implementation of problem-based learning in Asian medical schools and students' perceptions of their experience. Med Educ. 2003;37:401-9.

107. Prince KJAH, van Eijs PWLJ, Boshuizen HPA, van der Vleuten CPM, Scherpbier AJJA. General competencies of problem-based learning (PBL) and non-PBL graduates. Med Educ. 2005;39:394-401.

108. Dolmans DH, Schmidt HG. What drives the student in problem-based learning? Med Educ. 1994;28:372-80. 
109. Eagle CJ, Harasym PH, Mandin H. Effects of tutors with case expertise on problem-based learning issues. Acad Med. 1992;67:465-9.

110. Kamin C, O’Sullivan P, Deterding R, Younger M. A comparison of critical thinking in groups of third-year medical students in text, video, and virtual PBL case modalities. Acad Med. 2003;78:204-11.

111. Kelly PA, Haidet P, Schneider V, Searle N, Seidel CL, Richards BF. A comparison of inclass learner engagement across lecture, problem-based learning, and team learning using the STROBE classroom observation tool. Teach Learn Med. 2005;17:112-8.

112. Hendry GD, Ryan G, Harris J. Group problems in problem-based learning. Med Teach. 2003;25:609-16.

113. Charlin B, Mann K, Hansen P. The many faces of problem-based learning: a framework for understanding and comparison. Med Teach. 1998;20:323-30.

114. Bernstein P, Tipping J, Bercovitz K, Skinner HA. Shifting students and faculty to a PBL curriculum: attitudes changed and lessons learned. Acad Med. 1995;70:245-7.

115. Neville AJ. The problem-based learning tutor: teacher? facilitator? evaluator? Med Teach. 1999;21:393-401.

116. Thomas RE. Problem-based learning: measurable outcomes. Med Educ. 1997;31:320-9.

117. Blake RL, Hosokawa MC, Riley SL. Student performances on Step 1 and Step 2 of the United States Medical Licensing Examination following implementation of a problem-based learning curriculum. Acad Med. 2000;75:66-70.

118. Balslev T, de Grave WS, Muijtjens AMM, Scherpbier AJJA. Comparison of text and video cases in a postgraduate problem-based learning format. Med Educ. 2005;39:1086-92. 
119. Schmidt HG. Resolving inconsistencies in tutor expertise research: does lack of structure cause students to seek tutor guidance? Acad Med. 1994;69:656-62.

120. Distlehorst LH, Dawson E, Robbs RS, Barrows HS. Problem-based learning outcomes: the glass half-full. Acad Med. 2005;80:294-9.

121. Dornan T, Hadfield J, Brown M, Boshuizen H, Scherpbier A. How can medical students learn in a self-directed way in the clinical environment? Design-based research. Med Educ. 2005;39:356-64.

122. Dolmans DH, Gijselaers WH, Schmidt HG, van der Meer SB. Problem effectiveness in a course using problem-based learning. Acad Med. 1993;68:207-13.

123. Kassab S, Abu-Hijleh MF, Al-Shboul Q, Hamdy H. Student-led tutorials in problem-based learning: educational outcomes and students' perceptions. Med Teach. 2005;27:521-6.

124. Hill J, Rolfe IE, Pearson SA, Heathcote A. Do junior doctors feel they are prepared for hospital practice? a study of graduates from traditional and non-traditional medical schools. Med Educ. 1998;32:19-24. 